\title{
General Psychiatry Adapting behavioural interventions to compensate for cognitive dysfunction in persons with opioid use disorder
}

\author{
Colleen B Mistler (D) , ${ }^{1}$ Roman Shrestha, ${ }^{1}$ John Gunstad, ${ }^{2}$ Victoria Sanborn, ${ }^{2}$ \\ Michael M Copenhaver ${ }^{1}$
}

To cite: Mistler CB,

Shrestha R, Gunstad J, et al. Adapting behavioural interventions to compensate for cognitive dysfunction in persons with opioid use disorder. General Psychiatry 2021;34:e100412. doi:10.1136/ gpsych-2020-100412

Received 23 September 2020 Accepted 21 July 2021

\section{Check for updates}

\section{(c) Author(s) (or their} employer(s)) 2021. Re-use permitted under CC BY-NC. No commercial re-use. See rights and permissions. Published by BMJ.

${ }^{1}$ Allied Health Sciences, University of Connecticut, Storrs, Connecticut, USA

${ }^{2}$ Department of Psychology, Kent State University, Kent, Ohio, USA

Correspondence to

Colleen B Mistler;

colleen.mistler@uconn.edu

\section{ABSTRACT}

Treatment for opioid use disorder (OUD) is often in the context of biobehavioural interventions, consisting of medication for OUD (for example, methadone and buprenorphine), which is accompanied by psychoeducation and/or behavioural therapies. Patients with OUD often display weaknesses in cognitive function that may impact the efficacy of such behavioural interventions. A review of the literature was conducted to: (1) describe common cognitive dysfunction profiles among patients with OUD, (2) outline intervention approaches for patients with OUD, (3) consider the cognitive demands that interventions place on patients with OUD and (4) identify potential accommodation strategies that may be used to optimise treatment outcomes.

Cognitive profiles of patients with OUD often include weaknesses in executive function, attention, memory and information processing. Behavioural interventions require the patients' ability to learn, understand and remember information (placing specific cognitive demands on patients). Accommodation strategies are, therefore, needed for patients with challenges in one or more of these areas. Research on accommodation strategies for patients with OUD is very limited. We applied research from populations with similar cognitive profiles to form a comprehensive collection of potential strategies to compensate for cognitive dysfunction among patients with OUD. The cognitive profiles and accommodation strategies included in this review are intended to inform future intervention research aimed at improving outcomes among patients with OUD.

\section{INTRODUCTION}

The ongoing opioid epidemic is a public health crisis that has taken the lives of almost half a million people in the USA, as opioid use disorder (OUD) diagnoses and overdose rates have exponentially increased in the past 10 years. ${ }^{1}$ A wide range of comorbidities is common among persons with OUD. An understudied comorbidity among persons with OUD is cognitive dysfunction, commonly reported as deficits in executive functioning, attention, working memory and episodic memory. ${ }^{2-6}$ Cognitive dysfunction can dramatically impede drug treatment engagement and retention in care, resulting in poorer adherence to medications. ${ }^{78}$ It can also adversely impact motivation and behavioural skills that influence treatment outcomes. ${ }^{59}$ Treatment outcomes affected by cognitive dysfunction have been demonstrated to limit treatment compliance, including a reduced willingness to start and stay in treatment, low attendance at behavioural intervention sessions and reduced insight in regard to the benefits of treatment. ${ }^{10}$ This may be partially explained by poor behavioural intervention effects on patients with OUD, as the strategies used in behavioural interventions for OUD treatments may not be ideally tailored to meet the levels/forms of cognitive dysfunction among this population. ${ }^{11}$ Therefore, cognitive dysfunction and tailored accommodation strategies must be better understood and carefully matched in order to improve OUD treatment outcomes. ${ }^{12}$

Several behavioural intervention approaches are used among patients with OUD, including cognitive-behavioural therapy (CBT), contingency management $(\mathrm{CM})$, motivational interviewing (MI) and psychoeducation techniques. ${ }^{111314}$ While efficacious, these approaches also place significant cognitive demands on patients, ${ }^{14-16}$ which may limit their efficacy unless appropriate accommodation strategies are incorporated. ${ }^{17}$ In fact, the Substance Abuse and Mental Health Services Administration (SAMHSA) recently published the Treatment Improvement Protocol to provide treatment recommendations and accommodations for substance use disorder (SUD) treatment targeting people with cognitive dysfunction. ${ }^{17}$ The American Society of Addictive Medicine has also published treatment criteria with specific behavioural therapy accommodations for adult patients with cognitive dysfunction. ${ }^{18}$ Building on 
this foundation and relevant work with other patient populations, this review provides context by describing the cognitive features common to patients with OUD and the cognitive demands that interventions exert. However, our primary aim is to identify potential accommodation strategies to optimise treatment outcomes among patients with OUD for treatment providers. Here, we focus on compensatory accommodation strategies, as opposed to cognitive rehabilitation training that may require additional training, for ideal uptake and optimisation in a clinical drug treatment setting.

\section{COGNITIVE PROFILES AMONG PATIENTS WITH OUD}

Opioids interfere with brain functions, causing abnormal neuron activation. ${ }^{19}$ The rewards system of the brain (basal ganglia), decision-making system (prefrontal cortex), emotional response system (extended amygdala) and physical response system (brain stem) are all damaged with excessive opioid use. ${ }^{19}$ Patients receiving treatment for OUD tend to demonstrate diminished performance on cognitive testing. Cognitive dysfunction among patients with OUD in treatment often includes specific deficits in executive function, memory, attention and communication skills. ${ }^{20-22}$ Persons with OUD exhibit poorer decision-making and longer deliberation times, increasing their affective impulsivity. ${ }^{23} 24$ Inhibitory control is often impaired in persons with OUD, demonstrating the lack of ability to process negative feedback. ${ }^{20}$ Impaired semantic priming and verbal learning have also been observed in persons with OUD, revealing the inability of these individuals to recall structured information. ${ }^{25}$ Cognitive dysfunction has also been identified as a predictor of poor emotional perception among opioid-dependent individuals on medication for OUD (MOUD).$^{26}$ These cognitive profiles often result in unfavourable treatment outcomes, including poorer medication adherence, less treatment engagement and retention, and increased risk behaviours. ${ }^{78}$

SAMHSA recommends reviewing each patient's psychosocial history (eg, residential life, family, employment status and mental health) in combination with cognitive function screening to help develop patient profiles. Treatment providers should be aware of the degree to which cognitive dysfunction may affect a patient's life and inform treatment protocols. ${ }^{17}$ Bruijnen $e t a l^{27}$ used a combination of data, including medical history, demographics and testing, to examine cognitive function among patients enrolled in addiction treatment. They found distinct cognitive profiles among patients with SUDs. Opioid users showed lower scores on tasks of visuospatial abilities compared with users of other substances. Younger substance users showed better cognitive functioning than older individuals. ${ }^{27}$ Patient demographics and medical chart data have also been used to develop a cognitive dysfunction risk score among a sample of opioid-dependent individuals, demonstrating the potential to rapidly screen and predict levels of cognitive dysfunction in a clinical treatment setting. ${ }^{28}$

Sanborn $e t a l^{29}$ used a similar approach in identifying patient profiles among patients with OUD in addiction treatment. This neuropsychological assessment research incorporated the National Institute of Health Toolbox for the Assessment of Neurological Behavior and Function Cognition Battery and revealed two distinct profiles among patients with OUD: (1) those with significant impairment on tasks mediated by frontal brain regions (eg, attention and executive function) and (2) those with intact cognitive function. ${ }^{29}$ Those in the impaired group were also characterised as significantly older, more likely to be identified as an ethnic minority and less likely to use alcohol.

Other assessment studies regarding the identification of cognitive profiles among patients with OUD have incorporated the Brief Inventory of Neurocognitive Impairment $\mathrm{t}^{30}$ - a self-report measure of neurocognitive dysfunctions-and have identified three subgroups of patient profiles: (1) those with minimal cognitive dysfunction, (2) those with moderate symptoms of cognitive dysfunction including a history of head injury and (3) those with numerous symptoms of cognitive dysfunction across multiple subtest domains. Compared with patients in the minimal/moderate cognitive dysfunction subgroup, patients with numerous cognitive symptoms were found to have significantly elevated depressive symptoms, lower education levels and poorer performance on objective neuropsychological testing. ${ }^{30}$

Similar deficits have also been found on laboratorybased measures of cognitive function, as researchers show patients with OUD exhibit 'delayed discounting' (ie, decision-making that devalues delayed rewards and overvalues immediate rewards) as well as significantly more risky and impulsive decision-making. ${ }^{22} 2431$ This decision-making style is often associated with executive dysfunction and is consistent with the high impairment rates on similar tasks described above. Laboratorybased measures of the cognitive function used to assess persons with OUD have included structural and functional MRI and diffusion tensor imaging ${ }^{32}$; however, these assessment methods are not practical for application in a drug treatment setting owing to lack of time, training and proper equipment.

Taken in combination, a better understanding of common cognitive profiles among patients with OUD is an important step toward recognising barriers to their ability to fully benefit from treatment. This information may also guide the selection of appropriate accommodation strategies that can be incorporated into existing treatment approaches. The next and equally important step in this process involves identifying what features are most commonly used in intervention approaches for patients with OUD and considering what/the extent of the cognitive demands that popular approaches may place on patients with OUD. 


\section{BEHAVIOURAL APPROACHES CAN SUPPORT OUD TREATMENT}

The most common evidence-based medical treatment for OUD is MOUD, including methadone and buprenorphine. MOUD is a comprehensive treatment strategy that addresses the physiological basis of opioid dependence through opioid replacement therapy. ${ }^{33}$ MOUD, however, is not intended as a singular treatment for OUD, and several evidence-based behavioural strategies have been used concurrently to support MOUD. Common behavioural approaches include CBT, MI and CM. ${ }^{13} 1434$ Various psychoeducational counselling approaches are also provided in the context of MOUD. ${ }^{35}$ Such approaches are often used to increase knowledge, motivation and use of coping skills related to addiction and health risk reduction. ${ }^{36}$

\section{Cognitive-behavioural therapy}

CBT is an efficacious behavioural treatment used to enhance treatment outcomes among people with a range of disorders (eg, HIV, attention deficit hyperactivity disorder (ADHD), depression and anxiety) as well as SUD, ${ }^{34} 3637$ including OUD. ${ }^{35}{ }^{38}$ CBT follows three principles: (1) cognitive processes impact behaviour, (2) cognition can be altered and (3) behaviour change may be achieved through changes in cognitive processes. ${ }^{36} 38$ In the context of substance use treatment, a counsellor may use CBT to assist individuals in identifying high-risk situations, as well as developing strategies for coping with these high-risk situations to avoid relapse. ${ }^{34} 3637$ These coping strategies are typically both behavioural (avoid the people/places/things that trigger urges for opioid use) and cognitive (challenging thought patterns to counteract relapse cues) ${ }^{39}$ By practising skills during and between sessions, CBT can help a patient strengthen these skills to avoid relapse. ${ }^{13}$

\section{Contingency management}

$\mathrm{CM}$ is a well-established, evidence-based behavioural therapy that has been applied to a wide range of problems, including addiction and other health issues (eg, medication adherence and retention in treatment) ${ }^{13}$ among patients with OUD. This approach is rooted in operant conditioning, a learning process focused on positive reinforcement of desired behaviours. ${ }^{34} \mathrm{CM}$ approaches take into consideration the high rates of delayed discounting in people with OUD by providing immediate and tangible reinforcement that is 'contingent' on targeted behaviours (eg, abstinence from illicit drug use). Like 12-step programmes, CM provides praise for meeting short-term abstinence goals, a strategy that may maximise treatment outcomes as people with OUD may experience the immediate rewards as particularly satisfying. CM achieves its high success rate by shifting the perception of immediate rewards (ie, from the emotional/physical response of getting high to the gratifying feeling of being rewarded for abstinence). ${ }^{40} \mathrm{CM}$ has also been found to effectively improve treatment engagement among cognitively impaired individuals with OUD. ${ }^{41}$

\section{Motivational interviewing}

MI is another widely used evidence-based approach focused on enhancing behaviour change by increasing internal and external forms of motivation and selfefficacy. It is often implemented to help people with mental illness and SUD, including OUD ${ }^{42}$ and has also been shown to improve treatment retention rates. ${ }^{14} \mathrm{MI}$ uses a client-centred/collaborative approach between the clinician and the patient to increase intrinsic motivation and self-efficacy in order to change target behaviours. ${ }^{43}$ Four central principles of MI are the following: (1) express empathy, (2) increase awareness of discrepancies between undesirable behaviours and values inconsistent with those behaviours, (3) roll with resistance rather than confronting the patient directly and (4) support self-efficacy. In addition, MI clinicians employ decisional balance procedures to help clients weigh the pros and cons of change while using other MI skills to help tip the balance towards positive change. ${ }^{43}$ Research using an adapted MI approach has been positively associated with achieving therapeutic goals and negatively associated with the number of relapses and methadone dose in persons with OUD. ${ }^{44}$

\section{Psychoeducational approaches}

Addiction treatment programmes commonly employ psychoeducational approaches to inform participants about topics relevant to their addiction. Such approaches are often conducted in group 'workshop' style sessions with core components that include didactics, video presentations, pertinent role-playing situations and learning risk reduction skills, which patients are taught to apply to their real-life situations. A number of addiction-related psychoeducational approaches have been developed and tested under controlled conditions ${ }^{45}$ to address addiction, HIV prevention and awareness of HIV. As efficacious psychoeducational approaches are easily integrated into the drug treatment milieu, they are popular in community-based addiction programmes where many high-risk patients with OUD enrol in treatment. As discussed below, however, such approaches may be impeded by cognitive dysfunction among patients with OUD.

\section{COGNITIVE DEMANDS OF INTERVENTION APPROACHES USED FOR OUD}

Although intervention approaches similar to those outlined above are convenient to deliver in the context of MOUD, many of their respective intervention tasks are likely to tax the cognitive resources of patients with OUD (table 1). Such interventions have been widely implemented to treat various health conditions (eg, HIV, OUD, ADHD, post-traumatic brain injury (post-TBI) and depression) ${ }^{46-48}$ Though these health conditions often co-occur with cognitive dysfunction, ${ }^{49}{ }^{50}$ research on tailoring accommodation strategies to match cognitive dysfunction has been limited and, for the most part, 
Table 1 Cognitive dysfunction and intervention tasks impeded

\begin{tabular}{|c|c|c|}
\hline $\begin{array}{l}\text { Cognitive } \\
\text { domains }\end{array}$ & Intervention tasks impeded & Accommodation strategies \\
\hline $\begin{array}{l}\text { Executive function } \\
143856\end{array}$ & $\begin{array}{l}\text { Anticipating future consequences of } \\
\text { behaviour }^{1456} \\
\text { Executive planning skills }{ }^{16} 5758 \\
\text { Decision-making/decisional balance } \\
165758 \\
\text { Self-regulation } \\
\text { Logical reasoning }^{50}\end{array}$ & $\begin{array}{l}\text { Associating behaviour with situational cues (anticipating risky situations) }{ }^{6097} \\
\text { Consciously linking actions to a triggering cue (storytelling techniques using } \\
\text { imagery) } \\
\text { Planning (identifying and organising steps required to meet goal) }{ }^{1697} \\
\text { Valuing future events (recognising the benefits of drug treatment) }{ }^{16}\end{array}$ \\
\hline $\begin{array}{l}\text { Memory } \\
165054\end{array}$ & $\begin{array}{l}\text { Recalling reasons for past behaviour }{ }^{50} \\
\text { Remembering cues to reduce risk } \\
\text { behaviour }^{50} \\
\text { Recollecting information from previous } \\
\text { sessions } \\
\text { Learning new information } \\
50\end{array}$ & $\begin{array}{l}\text { Memory aids (reminders and cues to be used between sessions) }{ }^{38} 100 \\
\text { Example: therapy notebooks, electronic memory devices, SMS messages, } \\
\text { calendars and alarms }{ }^{385059686971-747677} \\
\text { Summarising and reiterating information (frequent review throughout sessions) } \\
97 \\
\text { Prospective memory (emphasising routine, developing cues and elaborating on } \\
\text { positive health behaviours) } \\
\text { Environmental engineering (preparing for adverse events) } \\
359799100\end{array}$ \\
\hline $\begin{array}{l}\text { Information } \\
\text { processing } \\
3854\end{array}$ & $\begin{array}{l}\text { Processing written materials }{ }^{15} \\
\text { Managing complex language } \\
\text { Accurate interpretation of feedback } \\
\text { Understanding the consequences of } \\
\text { behaviour }^{50}\end{array}$ & $\begin{array}{l}\text { Multimodal presentation of material (verbal, visual and hands-on) }{ }^{15} 385078-81 \\
\text { Simple language (clear, concrete examples aligned with the level of health } \\
\text { literacy) } \\
\text { Presenting information more slowly (allowing extra time for responses) } \\
83975065718082 \\
\text { Assessment with immediate feedback (oral and written clarification of material) } \\
85-89\end{array}$ \\
\hline
\end{tabular}

SMS, short message service.

has excluded patients with OUD. ${ }^{51}$ However, studies of other relevant patient populations have examined accommodation strategies to compensate for cognitive dysfunction in those patients (eg, ADHD and post-TBI) ${ }^{15} 354051-53$ which is integrated below.

In terms of specific cognitive requirements, CBT presumes that participants possess an intact memory, cognitive flexibility and the ability to accurately interpret counsellor feedback to execute preset plans in high-risk scenarios and anticipate/plan for future risks. ${ }^{54}$ Because this approach requires the anticipation of future actions, it demands substantial memory and executive function. The multifaceted components of CBT also require that patients appropriately allocate attention, spontaneously recall information and effectively communicate during and between sessions. ${ }^{38}$ CBT often incorporates written materials designed to assume that participants have intact cognitive ability and psychological flexibility. ${ }^{15}$ Significant impairment in verbal working memory and emotion regulation may also limit the efficacy of CBT. ${ }^{35} 55$

Like CBT, the efficacy of CM and MI approaches may also be limited by the cognitive capacity and ability to anticipate future consequences of target behaviours of patients with OUD. ${ }^{146} \mathrm{CM}$, for example, requires participants to successfully plan and make appropriate decisions while under pressure (ie, executive functioning), which are often demonstrated weaknesses in the cognitive profile of patients with OUD. ${ }^{16} 5758$ As previously described, CM uses immediate and tangible rewards for positive target behaviours ${ }^{34}$ to reinforce patients with OUD as they tend to display high rates of delayed discounting. ${ }^{22} 2431$ Owing to cognitive dysfunction, however, relapse may be more likely if the anticipated reward is not perceived as readily accessible. Additionally, the demands of $\mathrm{CM}$ on memory and cognitive flexibility may reduce its efficacy among a large portion of patients with OUD with cognitive dysfunction. ${ }^{16}$

Similarly, in MI and various psychoeducational approaches, many patients with OUD may struggle with hands-on exercises such as decisional balance demonstrations owing to the impulsive decision-making and delayed discounting characteristics that are common in patients with OUD. Further, MI requires sustained attention from the patients over an extended period. ${ }^{14}$ The basic cognitive abilities of attention, memory and motivation are needed to engage in behavioural treatment and are similar across intervention types. ${ }^{54}$ Accommodation strategies are, therefore, needed among patients with OUD with significant cognitive dysfunction. The following section summarises possible accommodation strategies to address cognitive dysfunction among patients with OUD to optimise their treatment outcomes. Such accommodation strategies have not yet been explicitly tested among patients with OUD. ${ }^{12} \mathrm{We}$ 
have, therefore, taken into consideration the similarities of cognitive profiles between patients with OUD and those studied across other health conditions ${ }^{15} 354051-53$ and have identified accommodation strategies found to be most applicable.

\section{ACCOMMODATION STRATEGIES FOR COGNITIVE DYSFUNCTION}

A series of restorative and compensatory strategies have been developed to address weaknesses in cognitive function. ${ }^{59} \mathrm{~A}$ restorative approach aims at reinforcing and strengthening impaired executive function through repeated skills training. Memory drills, reality orientation therapy and computer-assisted cognitive rehabilitation, for example, are restorative approaches used to improve these mental abilities ${ }^{59}$ to repair one's existing cognitive functioning. In contrast, executive functioning can also be improved through compensatory strategies, such as planning future events and consciously linking actions to behavioural cues. ${ }^{60}$

The current review focuses on compensatory approaches, fit for a clinical drug treatment setting, which involve bypassing or working around the specific area(s) of dysfunction rather than aiming to repair it. Compensatory strategies, for example, use techniques to improve one's likelihood of remembering (eg, memory aids) and ability to retain information (eg, multimodal presentation of information). ${ }^{51}{ }^{59}$ Frequent and consistent use of compensatory strategies has been associated with higher independent functioning among older adults with varying levels of cognitive impairment. ${ }^{51}$ These strategies address the cognitive barriers to engaging in treatment often present in people with OUD. ${ }^{1718}$

Patients with weaknesses in memory, attention and communication may benefit from more direct intervention content delivery and reinforcement. ${ }^{18}$ With minimal training, clinicians can use compensatory strategies to modify established behavioural approaches to support addiction treatment. ${ }^{17}$ Compensatory learning strategies (eg, shortening the length of sessions, distributing information across sessions, memory aids/reminders and using simple language) have been shown to improve treatment outcomes (eg, medication adherence and motivation) among people with cognitive impairment. ${ }^{5161}$ Most researches in this domain have been performed among people with cognitive profiles similar to those identified in persons with OUD, including people with ADHD or post-TBI. ${ }^{35} 38$ Given the parallels in cognitive profiles, it may be feasible to tailor the compensatory strategies outlined below, by cognitive domain, to optimise treatment outcomes among people with OUD.

\section{Addressing dysfunction on executive functioning}

Associate behaviours with situational cues

Anticipating risky situations and the future consequences of behaviour is a recommended strategy to address impulsive decision-making among persons with cognitive dysfunction. ${ }^{60}$ Planning for adverse events, in the context of high-risk environments, by identifying and recognising the steps needed to avoid the consequences of overt behaviours can accommodate executive dysfunction. ${ }^{16}$ This can be done by role-playing or providing patients with various case scenarios of risky situations and asking them to give examples of refusal skills for each particular scenario. Presenting a variety of high-risk situations and redirecting patients to focus on future-oriented tasks are recommended for those who may have difficulties planning and generating strategies for complex actions. ${ }^{62}$ Incorporating guided imagery into behavioural interventions and using story-telling techniques to help patients link actions to triggering cues help to compensate for impairments in executive functioning. ${ }^{14} 163860$

\section{Addressing dysfunction in attention and concentration Length and frequency of sessions}

Shortening the length of sessions is a recommended modification to behavioural interventions for people with cognitive dysfunction. Sessions that are less than 1 hour in length maximise the retention of information, particularly in those with attention deficits. ${ }^{1538}$ Similarly, distributed practice is a strategy that involves spreading intervention content across a greater number of sessions leading to less content being concentrated in each session. This has been found to increase participants' retention of the material. ${ }^{63}$ Increasing the frequency of sessions (more than once per week) also allows the patients to process and consolidate information over a greater number of days, which leads to a greater mastery of the material. ${ }^{18}$ More frequent meetings at the beginning of the treatment have been found to improve cognitive function and recovery from mental health disorders. ${ }^{1764}$ Acknowledging a patient's attentional abilities and working with the patient to develop strategies to increase attention to content are also recommended. ${ }^{17}$ An agreed on signal that the client may use to alert the clinician if/when their attention is fading can be useful in determining the optimal length of sessions. ${ }^{38}$

\section{Structure and consistency}

Highly structured and consistent sessions can also be used to help accommodate attentional deficits. ${ }^{38}$ To conduct a session that is structured and consistent, clinical staff need to be well organised and manage time well so that sessions begin and end on time and accomplish the prespecified objectives. ${ }^{50-67}$ Identifying the objectives of the session with the client also prepares the client for what is expected throughout the session. As such, having an agenda with objectives clarifies the purpose of each segment of the session. In group sessions, the counsellor can appoint a group member to serve as a timekeeper to help the group stay focused, on track and on time. This strategy provides clients with a sense of control, encourages responsibility for progressing on time and may help manage patients who have difficulty clearly and succinctly articulating their thoughts or whose speech tends to ramble or perseverate.$^{5065-67}$ Summarising and reporting 
key points at frequent intervals throughout a session also help refocus individuals and promote learning for those who have difficulty paying attention. ${ }^{68}$ A routine 'closure' at the end of each session can serve to reiterate previously learnt material and set the stage for content the patient should expect in the next session. ${ }^{36}$

\section{Addressing dysfunction in memory}

\section{Use of memory aids}

Memory aids offer a practical way to accommodate cognitive challenges not only during treatment sessions but also in real-world situations outside of treatment. Memory aids include reminders, cues and organisational aids that offer a convenient way to review information and skills between sessions and after completion of treatment when there is a much greater possibility of high-risk behaviour. Examples of memory aids include assistive technologies/electronic memory devices, calendars, alarms or reminders. ${ }^{38} 505968-74$ The use of mobile technologies (eg, basic mobile phones, patient monitoring devices, personal digital assistants and other wireless devices) offers a unique opportunity to support and provide assistance to individuals with cognitive impairment. ${ }^{75}$ Proactive planning of treatment-compatible activities (eg, using a memory book to track day and time that the patient will engage in a specific treatment-related activity such as attending support group), together with the necessary preparatory steps (eg, specifying when, where, how and with whom), can also enhance a patient's sense of control over daily events and facilitate action initiation. ${ }^{52}$

A therapy notebook is a useful tool for participants to review during the session and can be used to highlight important points to recall later. ${ }^{17}$ This strategy can be particularly crucial in the early stages of treatment when patients try to establish major changes to their daily routines that may initially feel overwhelming. ${ }^{5068-72} 76$ Routines gradually transfer into habits and can then be more easily stored in long-term memory. These habits serve as an internal memory strategy for participants once a routine has been established. Routines can also be converted to an external memory strategy using calendar reminders. ${ }^{77}$

\section{Prospective memory}

Prospective memory involves the ability to plan and successfully execute delayed intentions in the future. Interventions to improve prospective memory involve multiple strategies used to support key components of prospective remembering. Common examples related to medication adherence include: emphasising routines (same place and same medications), developing cues (linked with daily activities), converting time-based to event-based ('taking medication with breakfast', as opposed to 'at 08:00'), elaborating the action of taking medications (often in the context of distraction, saying aloud 'I took my medication'), doing it now (taking medicine immediately following the thought). Other examples include the use of a pill organiser (to provide a check and balance system), the use of implementation intention (consciously linking action to a cue, such as 'I need to take my medicine to get the benefits'), spaced retrieval (teach-wait-ask, ask again) and practice. ${ }^{60}$

\section{Environmental engineering}

Like prospective memory, environmental engineering involves setting up one's day in a manner that fully anticipates possible adverse events. The goal of environmental engineering is to institute systems designed to account for and work around cognitive dysfunction in everyday life. These systems, such as using a daily planner and/ or removing distractions from the workplace, act as a strategy to prevent external sources from overly influencing decision-making. ${ }^{35}$

\section{Addressing dysfunction in information processing Multimodal presentation of material}

Presenting treatment/intervention content using a range of modalities, including verbally, visually and experientially (ie, hands-on exercises), can stimulate information processing. ${ }^{15} 3850$ 78-81 For example, while verbally presenting the information (ie, 'talk therapy' style), key terms or concepts can be written on a flipchart or marker board, or simple diagrams can be drawn that patients can follow visually. Providing copies of printed handouts of material covered during intervention sessions may also aid in retaining information and skills. Live/interactive demonstrations can also help emphasise particular behavioural skills. Common demonstration activities that have been used in HIV prevention sessions, for example, include needle cleaning and condom application to a replica. The clinician can also show slides or video clips followed by discussion and critique of proper risk-reduction steps. Participants should be encouraged to actively engage in the treatment process by asking questions or volunteering to provide examples based on personal experiences. ${ }^{50} 6578-81$

\section{Use simple language and review material frequently}

Treatment counsellors should use clear, simple and basic language to communicate the material in order to facilitate learning and information processing. ${ }^{17}$ Frequent review of material and the most salient concepts can also enhance information processing and retention. ${ }^{15} 50657182-84$ A review can be provided at the beginning and in the middle of sessions (eg, revisiting key content previously covered) by referring back to the information outlined on flipcharts or presented in slides. If breaks are commonly used during longer intervention sessions, it may also be useful to recap what was covered before the break in order to re-establish the foundation and mindset for subsequent material. Similarly, at the close of a session, it may be helpful to provide an overall summary of the session and prepare participants for what to expect next. ${ }^{18} 5065718283$ Presenting information slowly during sessions and allowing extra time for participant responses can also enhance the quality of learning, ${ }^{38}$ as 
well as tailoring intervention content to be delivered in a patient's preferred mode of communication. ${ }^{15}$

\section{Assessment with immediate feedback}

Assessment with prompt feedback provides patients the opportunity to demonstrate their acquired knowledge and allows intervention staff to provide immediate clarification regarding information, concepts or skills that were misunderstood. ${ }^{50}$ 85-88 Assessment may involve directly observing patients' participation in experiential exercises. When clients demonstrate their knowledge and skills, intervention staff can provide feedback to correct any misunderstanding. Assessment can also be accomplished by ending sessions with a brief quiz-administered either orally or in the written format-that assesses the acquisition of basic concepts covered in the session. This also provides immediate feedback and additional clarification of material that may have been misunderstood ${ }^{505-89}$ to prevent participants from processing and retaining misunderstood information following the session.

\section{DISCUSSION AND FUTURE DIRECTIONS}

In this review, we outline an often overlooked comorbidity among patients with OUD: cognitive dysfunction. Although rarely a study focus, cognitive dysfunction is a common feature of patients with OUD when involved in tasks requiring executive functioning, attention, working memory and episodic memory. ${ }^{2-6}$ Cognitive dysfunction has been linked to disruptions in several treatment outcomes, including less treatment engagement, lower retention in care and poorer treatment adherence. ${ }^{78}$ Additionally, it has been linked with diminished motivation and acquisition of behavioural skills, which can also negatively impact a range of outcomes. ${ }^{59}$ Therefore, the current review sought to explore systematic ways to enhance treatment outcomes of patients with OUD by identifying a number of potential strategies to accommodate their cognitive dysfunctions. Included in this review is an overview of common cognitive profiles of people with OUD, strategies to rapidly assess cognitive functioning and tailored accommodation strategies that can be integrated to evidence-based behavioural interventions to maximise effectiveness in a drug treatment setting. These compensatory accommodation strategies specifically address the domains of cognitive dysfunction common among people with OUD and include various techniques to help patients pay attention, retain and remember information and reduce the engagement in risk behaviours. This review is among the first to seek strategies to enhance the treatment of patients with OUD with cognitive dysfunction by synthesising relevant information from several related perspectives.

Commonly, a biobehavioural approach is used in drug treatment settings to emphasise behaviour change and harm reduction, in combination with MOUD. ${ }^{90}$ Evidence-based treatment for OUD, including MOUD, does not explicitly incorporate cognitive screening and tailored accommodation strategies for various forms of cognitive dysfunction. ${ }^{91}$ In considering interdisciplinary research involving several other patient populations who experience cognitive dysfunction (eg, ADHD and post-TBI), ${ }^{15} 354051-53$ it appears feasible to develop a combined screening/accommodation method that allows treatment providers to rapidly identify cognitive dysfunction among patients with OUD and then systematically match accommodation strategies accordingly. Although some brief screening measures exist that can be used within community-based drug treatment settings, ${ }^{27} 3092$ to date, none have demonstrated the precision necessary for this purpose.

Future research should focus on developing a measure that targets both cognitive performance indicators and capture of pertinent background information that can be collectively used to identify cognitive profiles. The prevalence of various cognitive dysfunction in persons with OUD, and its consequential impact on treatment outcomes, calls for greater clinical attention to cognitive screening in treatment protocols. Ideally, a cognitive screener would identify different types of cognitive dysfunction and point toward an individualised approach to maximise treatment outcomes. However, there is currently insufficient evidence to support compensatory or restorative strategies to accommodate cognitive dysfunction in this population.

To address this gap in research, we recommend future researchers to examine a range of potential strategies that may be used to accommodate cognitive dysfunction among patients with OUD in the context of drug treatment settings where large numbers of these patients can be readily accessed.

This review provides examples of accommodation strategies that show promise in improving behavioural intervention for patients with OUD. Pilot feasibility studies are needed to test the utility of each of these strategies to advance this field of research. We suggest that future studies explore the efficacy of using these compensatory strategies on different domains and severity of cognitive dysfunction and OUD treatment outcomes. Furthermore, investigating the difference in efficacy between compensatory and restorative strategies is another avenue of research that could inform future OUD treatment protocols. It may be most efficient to use innovative methods such as a multiphase optimisation strategy, ${ }^{93}$ or sequential multiple assignment randomized trial, ${ }^{94}$ to test and refine individual accommodation strategies to match cognitive profiles rather than using a traditional approach of choosing strategies based solely on information such as informal hypotheses, post-hoc non-experimental analyses or clinical experience.

Future directions in the process of tailoring strategies for this risk group should, therefore, encompass two inter-related areas of inquiry, including (1) developing a rapid and precise cognitive screening tool capable of guiding the selection and integration of optimal treatment strategies among patients with OUD with cognitive 
dysfunction(s) and (2) testing the potential efficacy of multiple accommodation strategies that, alone or in some combination, can be effectively used among patients with OUD in the context of drug treatment. This foundation of research would benefit from identifying the specific accommodation strategies that are most useful for particular domains of cognitive dysfunction, based on rapid screening assessments. Building on our current knowledge, complementary research in each of these areas may propel the field forward and improve the treatment of patients with OUD.

\section{LIMITATIONS}

This review included a comprehensive, though not systematic, review of relevant literature on cognitive profiles and common behavioural interventions for people in treatment for OUD. Relatedly, we also focused on studies reporting behavioural outcomes rather than, for example, MRI data, positron emission tomography or electroencephalogram data, in order to maximise the homogeneity of measures and generalisability to behavioural intervention studies. ${ }^{95}{ }^{96}$ In an effort to identify potential accommodation strategies to be paired with commonly used behavioural interventions in persons with OUD, we attempted to match the cognitive demands (eg, memory, attention, cognitive flexibility, decision-making and emotional regulation) of such behavioural interventions with the cognitive impairments often identified in persons with OUD. However, as limited studies on specific cognitive demands and accommodation strategies for persons in treatment for OUD have been published, we drew on parallel research with other patient populations (eg, ADHD and post-TBI). ${ }^{15} 354051-53$ While patterns of cognitive dysfunction in other populations may not be fully generalisable to people in treatment for OUD, we attempted to focus on specific domains of cognitive dysfunction that are most applicable to those with OUD (eg, executive function, memory, attention and communication skills) to examine potential accommodation strategies to address those domains.

\section{CONCLUSIONS}

This review focuses on an understudied area of research regarding persons with OUD: matching cognitive profiles with specific treatment accommodations. We highlight the cognitive demands placed on patients with OUD receiving MOUD during common behavioural interventions and outline potential means for adjusting treatment strategies. Future work should aim to develop a screening measure that targets both cognitive performance indicators and capture of pertinent background information that can be collectively used to develop cognitive profiles that may predict the ability to engage in and benefit from various treatment strategies. ${ }^{11}$ Future research should also endeavour to tailor and test specific strategies to accommodate cognitive dysfunction among patients with OUD to determine how best to optimise treatment outcomes.

\section{Twitter Colleen B Mistler @ColleenMistler}

Contributors CBM, as the first author, developed, drafted and revised this manuscript with input from other authors. RS provided his expertise in OUD treatment and relevant content knowledge to add to this manuscript. He also contributed as an editor. JG provided his expertise in neuropsychology in clinical assessment research to add to this manuscript. He also contributed as an editor. VS provided her knowledge of neuropsychology and behavioural therapies to add to this manuscript. She also contributed as an editor. MMC, as the last author, added conceptual input and an overview of the review aims. This contributor also added his expertise and content knowledge for OUD treatment. He also contributed as an editor.

Funding This work was supported by grants from the National Institute on Drug Abuse for research (R01DA044867; K24DA051344 to MMC) and training (K01DA051346 to RS), and from the National Institute of Mental Health (T32MH074387) to CBM.

Competing interests None declared.

Patient consent for publication Not required.

Provenance and peer review Not commissioned; externally peer reviewed.

Open access This is an open access article distributed in accordance with the Creative Commons Attribution Non Commercial (CC BY-NC 4.0) license, which permits others to distribute, remix, adapt, build upon this work non-commercially, and license their derivative works on different terms, provided the original work is properly cited, appropriate credit is given, any changes made indicated, and the use is non-commercial. See: http://creativecommons.org/licenses/by-nc/4.0/.

ORCID iD

Colleen B Mistler http://orcid.org/0000-0002-8681-0252

\section{REFERENCES}

1 Centers for Disease Control and Prevention. Understanding the Epidemic, 2020. Available: https://www.cdc.gov/drugoverdose/ epidemic/index.html

2 Rapeli P, Kivisaari R, Autti T, et al. Cognitive function during early abstinence from opioid dependence: a comparison to age, gender, and verbal intelligence matched controls. BMC Psychiatry 2006;6:9

3 Verdejo-García A, López-Torrecillas F, Giménez CO, et al. Clinical implications and methodological challenges in the study of the neuropsychological correlates of cannabis, stimulant, and opioid abuse. Neuropsychol Rev 2004;14:1-41.

4 Schiltenwolf M, Akbar M, Hug A, et al. Evidence of specific cognitive deficits in patients with chronic low back pain under longterm substitution treatment of opioids. Pain Physician 2014;17:9-20.

5 Anand P, Springer SA, Copenhaver MM, et al. Neurocognitive impairment and HIV risk factors: a reciprocal relationship. AIDS Behav 2010;14:1213-26.

6 Baldacchino A, Balfour DJK, Passetti F, et al. Neuropsychological consequences of chronic opioid use: a quantitative review and meta-analysis. Neurosci Biobehav Rev 2012;36:2056-68.

7 Altice FL, Kamarulzaman A, Soriano VV, et al. Treatment of medical, psychiatric, and substance-use comorbidities in people infected with HIV who use drugs. Lancet 2010;376:367-87.

8 Kamarulzaman A, Altice FL. Challenges in managing HIV in people who use drugs. Curr Opin Infect Dis 2015;28:10-16.

9 Huedo-Medina TB, Shrestha R, Copenhaver M. Modeling a Theory-Based approach to examine the influence of neurocognitive impairment on HIV risk reduction behaviors among drug users in treatment. AIDS Behav 2016;20:1646-57.

10 Rezapour T, Hatami J, Farhoudian A. Cognitive rehabilitation for individuals with opioid use disorder: a randomized controlled trial. Neuropsychol Rehabil 2019;29:1273-89.

11 Tsui CM, Li D. Substance abuse rehabilitation needs to be guided by well-developed practice model(s). Gen Psychiatr 2020;33:e100220.

12 Ezeabogu I, Copenhaver MM, Potrepka J. The influence of neurocognitive impairment on HIV treatment outcomes among drug-involved people living with HIV/AIDS. AIDS Care 2012;24:386-93. 
13 Carroll KM. Lost in translation? Moving contingency management and cognitive behavioral therapy into clinical practice. Ann N Y Acad Sci 2014;1327:94-111.

14 Madson MB, Schumacher JA, Baer JS, et al. Motivational interviewing for substance use: mapping out the next generation of research. J Subst Abuse Treat 2016;65:1-5.

15 Rossiter R, Holmes S. Access all areas: creative adaptations for CBT with people with cognitive impairments - illustrations and issues. The Cognitive Behaviour Therapist 2013;6.

16 Bickel WK, Jarmolowicz DP, Mueller ET, et al. Are executive function and impulsivity antipodes? A conceptual reconstruction with special reference to addiction. Psychopharmacology 2012;221:361-87.

17 Substance Abuse and Mental Health Services Administration. TIP 29: Substance Use Disorder Treatment for People With Physical and Cognitive Disabilities, 2012. Available: https://store.samhsa.gov/ product/TIP-29-Substance-Use-Disorder-Treatment-for-PeopleWith-Physical-and-Cognitive-Disabilities/SMA12-4078

18 David ML. ASAM criteria: treatment criteria for addictive, substancerelated, and co-occurring conditions. Third edn. Rockville: American society of addictive medicine, 2013.

19 National Institute on Drug Abuse. Drugs, Brains, and Behavior:The Science of Addiction, 2020. Available: https://www.drugabuse.gov/ publications/drugs-brains-behavior-science-addiction/drugs-brain

20 Ersche KD, Sahakian BJ. The neuropsychology of amphetamine and opiate dependence: implications for treatment. Neuropsychol Rev 2007;17:317-36.

21 Rass O, Schacht RL, Buckheit K, et al. A randomized controlled trial of the effects of working memory training in methadone maintenance patients. Drug Alcohol Depend 2015;156:38-46.

22 Kirby KN, Petry NM. Heroin and cocaine abusers have higher discount rates for delayed rewards than alcoholics or non-drugusing controls. Addiction 2004:99:461-71.

23 Fishbein DH, Krupitsky E, Flannery BA, et al. Neurocognitive characterizations of Russian heroin addicts without a significant history of other drug use. Drug Alcohol Depend 2007:90:25-38.

24 Verdejo-García A, Pérez-García M. Profile of executive deficits in cocaine and heroin polysubstance users: common and differential effects on separate executive components. Psychopharmacology 2007; 190:517-30.

25 Battistella S, Constantinou N, Morgan CJA, et al. Semantic priming and verbal learning in current opiate users, ex-users and non-user controls. Hum Psychopharmacol 2012;27:499-506.

26 McDonald S, Darke S, Kaye S, et al. Deficits in social perception in opioid maintenance patients, abstinent opioid users and non-opioid users. Addiction 2013;108:566-74.

27 Bruijnen CJWH, Dijkstra BAG, Walvoort SJW, et al. Prevalence of cognitive impairment in patients with substance use disorder. Drug Alcohol Rev 2019;38:435-42.

28 Copenhaver MM, Sanborn V, Shrestha R, et al. Developing a cognitive dysfunction risk score for use with opioid-dependent persons in drug treatment. Drug Alcohol Depend 2021;224:108726.

29 Sanborn V, Gunstad J, Shrestha R, et al. Cognitive profiles in persons with opioid use disorder enrolled in methadone treatment. Appl Neuropsychol 2020;19:1-7.

30 Gunstad J, Sanborn V, Shrestha R. Cluster analysis of data from the brief inventory of neurocognitive impairment (BINI) to develop Neuro-Cognitive profiles among opioid-dependent patients in drug treatment. Frontiers in Psychiatry. In press.

31 Ahn W-Y, Vassileva J. Machine-learning identifies substancespecific behavioral markers for opiate and stimulant dependence. Drug Alcohol Depend 2016;161:247-57.

32 Upadhyay J, Maleki N, Potter J, et al. Alterations in brain structure and functional connectivity in prescription opioid-dependent patients. Brain 2010;133:2098-114.

33 Substance Abuse and Mental Health Services Administration. Medication-assisted treatment of opioid use disorder, 2016. Available: https://www.samhsa.gov/medication-assisted-treatment

34 McHugh RK, Hearon BA, Otto MW. Cognitive behavioral therapy for substance use disorders. Psychiatr Clin North Am 2010;33:511-25.

35 Ramsay JR. CBT for adult ADHD: adaptations and Hypothesized mechanisms of change. J Cogn Psychother 2010;24:37-45.

36 Jhanjee S. Evidence based psychosocial interventions in substance use. Indian J Psychol Med 2014;36:112-8.

37 Chen J, Qian M, Sun C, et al. Clinical effectiveness of cognitive behavioural therapy on alcohol-dependent patients: an observation with the WeChat platform. Gen Psychiatr 2019;32:e100087.

38 Gallagher M, McLeod HJ, McMillan TM. A systematic review of recommended modifications of $\mathrm{CBT}$ for people with cognitive impairments following brain injury. Neuropsychol Rehabil 2019;29:1-21.
39 Sofuoglu M, DeVito EE, Waters AJ, et al. Cognitive enhancement as a treatment for drug addictions. Neuropharmacology 2013;64:452-63.

40 Bickel WK, Miller ML, Yi R, et al. Behavioral and neuroeconomics of drug addiction: competing neural systems and temporal discounting processes. Drug Alcohol Depend 2007;90 Suppl 1:S85-91.

41 Kiluk BD, Buck MB, Devore KA, et al. Performance-Based contingency management in cognitive remediation training: a pilot study. J Subst Abuse Treat 2017;72:80-8.

42 Substance Abuse and Mental Health Services Administration. SAMHSA-HRSA center for integrated health solutions (CIHS), 2017. Available: https://www.samhsa.gov/integrated-health-solutions

43 Miller WR, Rollnick S. Motivational interviewing: helping people change. 3rd edn. New York, NY: Guilford press, 2012.

44 Ziaee SS, Fadardi JS, Cox WM, et al. Effects of attention control training on drug abusers' attentional bias and treatment outcome. $J$ Consult Clin Psychol 2016;84:861-73.

45 Johnson SM, Naidoo AV. A psychoeducational approach for prevention of burnout among teachers dealing with HIV/AIDS in South Africa. AIDS Care 2017;29:73-8.

46 Centers for Disease Control and Prevention. Effective interventions, 2020. Available: https://www.cdc.gov/hiv/effective-interventions/ index.html

47 Centers for Disease Control and Prevention. Attention deficit / hyperactivity disorder, 2020. Available: https://www.cdc.gov/ ncbddd/adhd/behavior-therapy.htm

48 Webb CA, Auerbach RP, Derubeis RJ. Processes of change in CBT of adolescent depression: review and recommendations. J Clin Child Adolesc Psychol 2012;41:654-65.

49 National Institute on Drug Abuse. Comorbidity: substance use disorders and other mental illnesses drugfacts, 2018. https://www. drugabuse.gov/publications/drugfacts/comorbidity-substance-usedisorders-other-mental-illnesses

50 Copenhaver M, Avants SK, Warburton LA, et al. Intervening effectively with drug abusers infected with HIV: taking into account the potential for cognitive impairment. J Psychoactive Drugs 2003;35:209-18.

51 Tomaszewski Farias S, Schmitter-Edgecombe M, Weakley A, et al. Compensation strategies in older adults: association with cognition and everyday function. Am J Alzheimers Dis Other Demen 2018;33:184-91.

52 Kysow K, Park J, Johnston C. The use of compensatory strategies in adults with ADHD symptoms. Atten Defic Hyperact Disord 2017;9:73-88.

53 Canela C, Buadze A, Dube A, et al. Skills and compensation strategies in adult ADHD - A qualitative study. PLoS One 2017;12:e0184964.

54 Perry CJ, Lawrence AJ. Addiction, cognitive decline and therapy: seeking ways to escape a vicious cycle. Genes Brain Behav 2017:16:205-18.

55 Liu Y, Bi T, Zhang B, et al. Face and object visual working memory deficits in first-episode schizophrenia correlate with multiple neurocognitive performances. Gen Psychiatr 2021;34:e100338.

56 McClure SM, Bickel WK. A dual-systems perspective on addiction: contributions from neuroimaging and cognitive training. Ann NY Acad Sci 2014:1327:62-78.

57 Radu PT, Yi R, Bickel WK, et al. A mechanism for reducing delay discounting by altering temporal attention. J Exp Anal Behav 2011:96:363-85.

58 Poudel R, Riedel MC, Salo T, et al. Common and distinct brain activity associated with risky and ambiguous decision-making. Drug Alcohol Depend 2020;209:107884.

59 Barman A, Chatterjee A, Bhide R. Cognitive impairment and rehabilitation strategies after traumatic brain injury. Indian J Psychol Med 2016;38:172-81.

60 Insel KC, Einstein GO, Morrow DG, et al. A multifaceted prospective memory intervention to improve medication adherence: design of a randomized control trial. Contemp Clin Trials 2013;34:45-52.

61 Simon SS, Yokomizo JE, Bottino CMC. Cognitive intervention in amnestic mild cognitive impairment: a systematic review. Neurosci Biobehav Rev 2012;36:1163-78.

62 McDonald BC, Flashman LA, Saykin AJ. Executive dysfunction following traumatic brain injury: neural substrates and treatment strategies. NeuroRehabilitation 2002;17:333-44.

63 Cepeda NJ, Pashler H, Vul E, et al. Distributed practice in verbal recall tasks: a review and quantitative synthesis. Psychol Bull 2006;132:354-80.

64 Tiemens B, Kloos M, Spijker J, et al. Lower versus higher frequency of sessions in starting outpatient mental health care and the risk 
of a chronic course; a naturalistic cohort study. BMC Psychiatry 2019;19:228.

65 Margolin A, Avants SK, Warburton LA, et al. A randomized clinical trial of a manual-guided risk reduction intervention for HIV-positive injection drug users. Health Psychol 2003;22:223-8.

66 Safren SA, Otto MW, Sprich S, et al. Cognitive-behavioral therapy for ADHD in medication-treated adults with continued symptoms. Behav Res Ther 2005;43:831-42.

67 Knouse LE, Safren SA. Current status of cognitive behavioral therapy for adult attention-deficit hyperactivity disorder. Psychiatr Clin North Am 2010;33:497-509.

68 Gillespie A, Best C, O'Neill B. Cognitive function and assistive technology for cognition: a systematic review. J Int Neuropsychol Soc 2012;18:1-19.

69 Frank Lopresti E, Mihailidis A, Kirsch N. Assistive technology for cognitive rehabilitation: state of the art. Neuropsychol Rehabil 2004:14:5-39.

70 Oriani M, Moniz-Cook E, Binetti G, et al. An electronic memory aid to support prospective memory in patients in the early stages of Alzheimer's disease: a pilot study. Aging Ment Health 2003;7:22-7.

71 Harvey AG, Lee J, Williams J, et al. Improving outcome of psychosocial treatments by enhancing memory and learning. Perspect Psychol Sci 2014;9:161-79.

72 Bauer S, Okon E, Meermann R, et al. Technology-enhanced maintenance of treatment gains in eating disorders: efficacy of an intervention delivered via text messaging. J Consult Clin Psychol 2012;80:700-6.

73 Shrestha R, Altice FL, DiDomizio E, et al. Feasibility and acceptability of an mHealth-Based approach as an HIV prevention strategy among people who use drugs on pre-exposure prophylaxis. Patient Prefer Adherence 2020;14:107-18.

74 Shrestha R, Karki P, Copenhaver M. Interest in use of mHealth technology in HIV prevention and associated factors among highrisk drug users enrolled in methadone maintenance program. AIDS Care 2017;29:1144-8.

75 Bateman DR, Srinivas B, Emmett TW, et al. Categorizing health outcomes and efficacy of mHealth Apps for persons with cognitive impairment: a systematic review. J Med Internet Res 2017;19:e301-e.

76 Thickpenny-Davis KL, Barker-Collo SL. Evaluation of a structured group format memory rehabilitation program for adults following brain injury. J Head Trauma Rehabil 2007;22:303-13.

77 Bourgeois MS. Therapy techniques for mild cognitive impairment. Perspect Neurophysiol Neurogenic Speech Lang Disord 2013;23:1-12.

78 Cao Y, Theune M, Nijholt A. Towards Cognitive-Aware Multimodal Presentation: The Modality Effects in High-Load HCl. In: Harris $\mathrm{D}$, ed. Engineering psychology and cognitive ergonomics: 8th International Conference, EPCE 2009, held as part of $\mathrm{HCl}$ international 2009, San Diego, Ca, USA, July 19-24, 2009 proceedings. Berlin, Heidelberg: Springer Berlin Heidelberg, 2009: 3-12.

79 Mayer RE, Moreno R. Nine ways to reduce cognitive load in multimedia learning. Educ Psychol 2003;38:43-52.

80 Ginns P. Meta-Analysis of the modality effect. Learn Instr 2005:15:313-31.

81 Deubel P. An investigation of behaviorist and cognitive approaches to instructional multimedia design. JEMH 2003;12:63-90.
82 Shepherd L, Salkovskis PM, Morris M. Recording therapy sessions: an evaluation of patient and therapist reported behaviours, attitudes and preferences. Behav Cogn Psychother 2009;37:141-50.

83 Pratt SI, Mueser KT, Bartels SJ, et al. The impact of skills training on cognitive functioning in older people with serious mental illness. Am $J$ Geriatr Psychiatry 2013;21:242-50.

84 Gates N, Valenzuela M. Cognitive exercise and its role in cognitive function in older adults. Curr Psychiatry Rep 2010;12:20-7.

85 Ancelin ML, Artero S, Portet F, et al. Non-degenerative mild cognitive impairment in elderly people and use of anticholinergic drugs: longitudinal cohort study. BMJ 2006;332:455-9.

86 Epstein ML, Lazarus AD, Calvano TB, et al. Immediate feedback assessment technique promotes learning and corrects inaccurate first responses. Psychol Rec 2002;52:187-201.

87 Hattie J, Timperley $\mathrm{H}$. The power of feedback. Rev Educ Res 2007;77:81-112.

88 Tam S-F, Man W-K. Evaluating computer-assisted memory retraining programmes for people with post-head injury amnesia. Brain Inj 2004;18:461-70.

89 Patterson TL, Bucardo J, McKibbin CL, et al. Development and pilot testing of a new psychosocial intervention for older Latinos with chronic psychosis. Schizophr Bull 2005;31:922-30.

90 National Institute on Drug Abuse. Principles of drug addiction treatment: a research-based guide. Third edn, 2018. https://www. drugabuse.gov/publications/principles-drug-addiction-treatmentresearch-based-guide-third-edition/evidence-based-approachesto-drug-addiction-treatment/behavioral-therapies

91 McCarty D, Priest KC, Korthuis PT. Treatment and prevention of opioid use disorder: challenges and opportunities. Annu Rev Public Health 2018;39:525-41.

92 Sanborn V, Gunstad J, Shrestha R, et al. Cognitive profiles in persons with opioid use disorder enrolled in methadone treatment. Appl Neuropsychol Adult 2020:1-7.

93 Collins LM, Murphy SA, Nair VN, et al. A strategy for optimizing and evaluating behavioral interventions. Ann Behav Med 2005;30:65-73.

94 Murphy SA, Lynch KG, Oslin D, et al. Developing adaptive treatment strategies in substance abuse research. Drug Alcohol Depend 2007;88 Suppl 2:S24-30

95 Smallwood RF, Potter JS, Robin DA. Neurophysiological mechanisms in acceptance and commitment therapy in opioidaddicted patients with chronic pain. Psychiatry Res Neuroimaging 2016:250:12-14.

96 Maclin JMA, Wang T, Xiao S. Biomarkers for the diagnosis of Alzheimer's disease, dementia Lewy body, frontotemporal dementia and vascular dementia. Gen Psychiatr 2019;32:e100054.

97 Virta M, Vedenpää A, Grönroos N, et al. Adults with ADHD benefit from cognitive-behaviorally oriented group rehabilitation: a study of 29 participants. J Atten Disord 2008;12:218-26.

98 Cuijpers P, Huibers M, Ebert DD, et al. How much psychotherapy is needed to treat depression? A metaregression analysis. J Affect Disord 2013;149:1-13.

99 Solanto MV, Marks DJ, Mitchell KJ, et al. Development of a new psychosocial treatment for adult ADHD. J Atten Disord 2008:11:728-36.

100 Rostain AL, Ramsay JR. A combined treatment approach for adults with ADHD--results of an open study of 43 patients. J Atten Disord 2006;10:150-9.

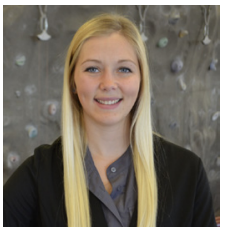

Colleen B. Mistler obtained a Master of Science degree from Springfield College in the USA, with a concentration in Health Promotion and Disease Prevention, in 2019. She is currently in her second year of a Doctor of Philosophy degree program in Health Promotion Sciences at the University of Connecticut. Colleen's education and research is funded by a National Institute of Mental Health T-32 training grant. She works closely with her advisor and mentor (Michael Copenhaver and Roman Shrestha) researching harm reduction among people in treatment for opioid use disorder. Colleen's main research interests include opioid use prevention, behavioral interventions for substance use treatment, HIV prevention and health equity. 